\title{
Probing deep level centers in GaN epilayers with variable-frequency capacitance-voltage characteristics of Au/GaN Schottky contacts
}

\author{
R. X. Wang, ${ }^{\text {a) }}$ S. J. Xu, ${ }^{\text {b) }}$ S. L. Shi, C. D. Beling, and S. Fung \\ Department of Physics, The University of Hong Kong, Pokfulam Road, Hong Kong, China \\ D. G. Zhao and H. Yang \\ State Key Laboratory on Integrated Optoelectronics, Institute of Semiconductors, Chinese Academy of \\ Sciences, Beijing 100083, China \\ X. M. Tao \\ Institute of Textiles and Clothing, The Hong Kong Polytechnic University, Kowloon, Hong Kong, China
}

(Received 3 July 2006; accepted 10 August 2006; published online 3 October 2006)

\begin{abstract}
Under identical preparation conditions, $\mathrm{Au} / \mathrm{GaN}$ Schottky contacts were prepared on two kinds of GaN epilayers with significantly different background electron concentrations and mobility as well as yellow emission intensities. Current-voltage $(I-V)$ and variable-frequency capacitance-voltage $(C-V)$ characteristics show that the Schottky contacts on the GaN epilayer with a higher background carrier concentration and strong yellow emission exhibit anomalous reverse-bias $I-V$ and $C$ - $V$ characteristics. This is attributed to the presence of deep level centers. Theoretical simulation of the low-frequency $C$ - $V$ curves leads to a determination of the density and energy level position of the deep centers. (C) 2006 American Institute of Physics. [DOI: 10.1063/1.2358207]
\end{abstract}

In the past decade, GaN and related III-nitride compounds have attracted a great deal of interest because of their wide optoelectronic and electronic applications, such as blue/ ultraviolet light-emitting diodes, ultraviolet detectors, laser diodes, and high-temperature/high-power transistors. MetalGaN contacts including Schottky contacts are of great importance for device applications of GaN-based materials. Despite the commercialization of GaN-based bright lightemitting diodes and even laser diodes, ${ }^{1}$ a number of issues related to metal-GaN contacts (especially Schottky contacts) still remain. These are not well understood and are thus being actively investigated. ${ }^{2-8}$ Having a work function of $\sim 5.1 \mathrm{eV}$, gold $(\mathrm{Au})$ is one of the most used metals in the fabrication of metal-semiconductor Schottky and Ohmic contacts. For $\mathrm{Au} / \mathrm{GaN}$ Schottky contacts, several groups have reported their results. ${ }^{2,3,5}$ However, few studies have been reported to investigate the influence of deep level centers (which are closely associated with the yellow emission band) on the capacitance-voltage characteristics of metalGaN Schottky contacts. In this letter, we show experimentally that the deep level centers located at about $1.3 \mathrm{eV}$ can lead to typical anomalous reverse-bias $C-V$ characteristics of $\mathrm{Au} / \mathrm{GaN}$ Schottky diodes, particularly the low-frequency $C$ - $V$ characteristics.

Two $n$-type GaN epilayers, namely, S1 and S2, grown on (0001) sapphire substrate by metal organic chemical vapor deposition, were used as the starting materials for the study. Using standard Hall measurements, the electron concentration and mobility of samples $\mathrm{S} 1$ and $\mathrm{S} 2$ at room temperature were determined to be $1.9 \times 10^{17} \mathrm{~cm}^{-3}$ and $331 \mathrm{~cm}^{2} \mathrm{~V}^{-1} \mathrm{~s}^{-1}$, as well as $3.3 \times 10^{16} \mathrm{~cm}^{-3}$ and $836 \mathrm{~cm}^{2} \mathrm{~V}^{-1} \mathrm{~s}^{-1}$, respectively. Prior to making metal contacts, the samples were cleaned by immersing the samples sequentially in boiling acetone and

\footnotetext{
${ }^{a}$ Present address: Institute of Textiles and Clothing, Hong Kong Polytechnic University, Hung Hom, Kowloon, Hong Kong, China.

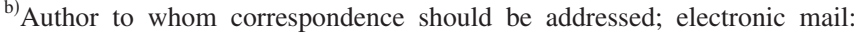
sjxu@hkucc.hku.hk
}

ethanol and de-ionized water. To remove any native oxide and contamination, they were dipped into different acid solutions after which the samples were rinsed in de-ionized water and dried with $\mathrm{N}_{2}$. Au and indium (In) were used to make Schottky and Ohmic contacts, respectively. $I-V$ measurements were carried out using an HP 4155A parameter analyzer, and frequency-dependent $C$ - $V$ characteristics of the Schottky contacts were measured using an HP 4294A parameter analyzer. For the photoluminescence (PL) measurements, the samples were excited by the $325 \mathrm{~nm}$ line of a Kimmon $\mathrm{He}-\mathrm{Cd}$ continuous-wave laser with an output power of $34 \mathrm{~mW}$ at room temperature. The PL setup has been described in detail elsewhere. ${ }^{9}$

Figure 1 shows typical room-temperature $I-V$ characteristics of the $\mathrm{Au} / \mathrm{GaN}$ Schottky contacts fabricated on the $\mathrm{S} 1$ and $\mathrm{S} 2 \mathrm{GaN}$ wafers. The $I-V$ curves clearly exhibit rectification behavior characteristic of common metal-semiconductor junctions. However, the reverse-bias characteristics of the

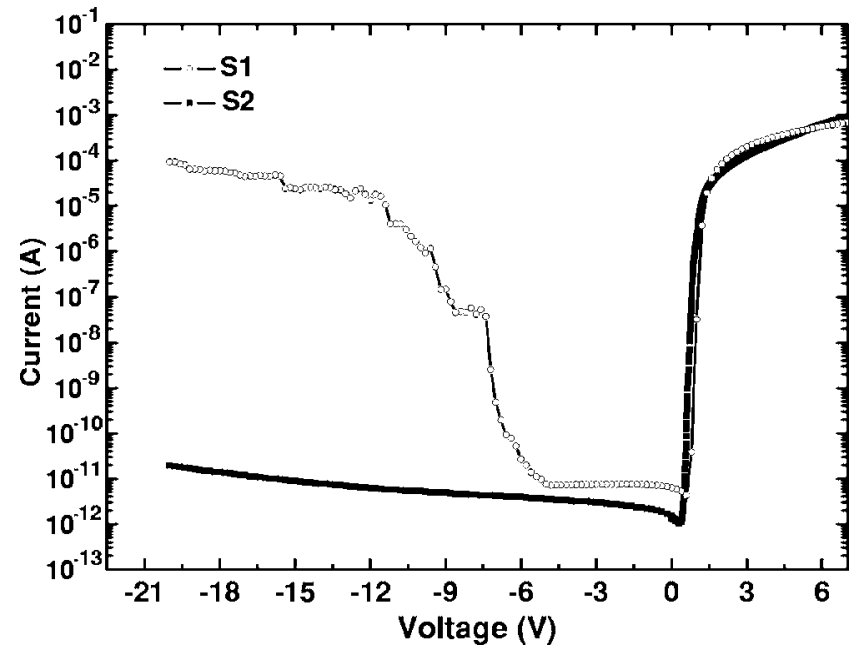

FIG. 1. Typical $I-V$ characteristics of the Au/GaN Schottky contacts fabricated on the S1 and S2 GaN epilayers. 

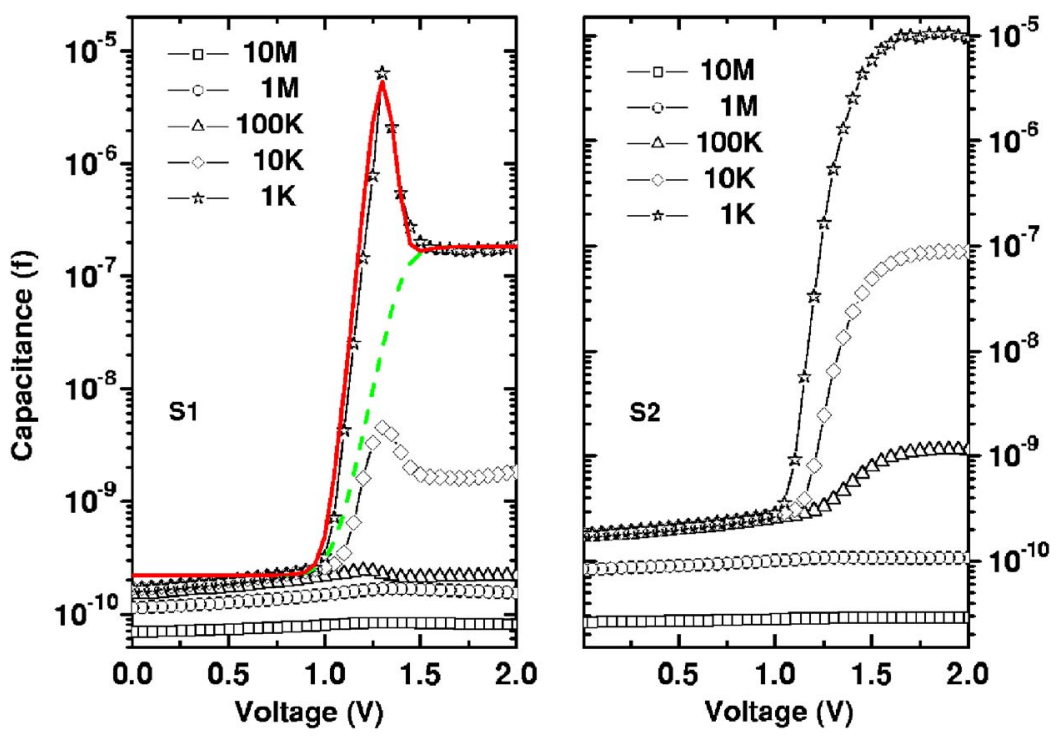

FIG. 2. (Color online) Frequency-dependent $C-V$ curves of both $\mathrm{Au}-\mathrm{GaN}$ Schottky contacts. The peak structure in the $C$ - $V$ curves of the gold Schottky contact on the $\mathrm{S} 1 \mathrm{GaN}$ epilayer is due to the surface states induced by the deep level centers. The solid line is the fitting curve using Eqs. (2)-(6) while the dashed line is the differential space-charge capacitance.

two kinds of Schottky contacts are very different. For the contacts fabricated on $\mathrm{S} 2$, their reverse-bias characteristics are not only uniform but also reproducible and stable. The reverse-bias leakage current is around $10^{-11} \mathrm{~A}$ at $-20 \mathrm{~V}$. In contrast, the contacts fabricated on S1 show a much larger reverse leakage current and lower breakdown voltage. The reproducibility and stability of the reverse-bias $I-V$ characteristics of the devices are poor. Based on the thermionicemission theory, the Schottky parameters can be extracted by fitting the forward exponential section of the $I-V$ characteristic using the equation ${ }^{10}$

$$
I=A A^{*} T^{2} e^{-q \phi_{b} / k T}\left[e^{q V / n k T}-1\right],
$$

where $A, A^{*}, \phi_{b}$, and $n$ are the contact area, Richardson constant, Schottky barrier height, and the ideality factor, respectively. The extracted average barrier heights $\phi_{b}$ of $\mathrm{Au} / \mathrm{GaN}$ contacts fabricated on $\mathrm{S} 1$ and $\mathrm{S} 2$ are 0.77 and $0.72 \mathrm{eV}$, respectively, whereas their average ideality factors $n$ are 1.45 and 1.73 , respectively. The values of $\phi_{b}$ and $n$ of the contacts fabricated on S1 look better than those on S2.

Now we turn to the $C-V$ characteristics of the devices. It is well known that defects strongly affect the electrical characteristics of metal-semiconductor junctions, in particular, the capacitance-voltage characteristics of the junctions. ${ }^{10}$ In fact, the effect of defects (especially deep traps) on the capacitance of a metal-semiconductor Schottky contact affords a very convenient method for detecting and characterizing an extremely low concentration of defects. ${ }^{11,12}$ Figure 2 shows the frequency-dependent $C-V$ curves of Au-GaN Schottky contacts fabricated on $\mathrm{S} 1$ and $\mathrm{S} 2$. It can be observed that the frequency-dependent $C$ - $V$ curves of both the samples are quite different from each other. The most noteworthy difference is that a peak structure around $1.3 \mathrm{~V}$ appears which becomes more prominent with decreasing applied frequency for the capacitance $\left(C_{\mathrm{S} 1}\right)$ of the device fabricated on $\mathrm{S} 1$. This is a typical $C-V$ characteristic of metal-semiconductor Schottky contact due to the existence of defect centers on semiconductor surface. ${ }^{12}$ In sharp contrast to the case of S1, the capacitance $\left(C_{\mathrm{S} 2}\right)$ of the devices fabricated on S2 simply and monotonically decreases when the applied voltage is lower than $\sim 1.3 \mathrm{~V}$, which is the typical behavior of an ideal metal-semiconductor Schottky contact without surface states. The simple monotonic decrease of the device capacitance is due to the variation of the space-charge capacitance with applied voltage. For the device with the surface states, an additional surface-state capacitance should be considered. Thus, the total capacitance of the device is written as

$$
C_{S}=C_{\mathrm{SS}}+C_{\mathrm{SC}}
$$

where $C_{\mathrm{SC}}$ and $C_{\mathrm{SS}}$ are, respectively, the differential spacecharge and surface-state capacitances. According to the work of Many et al., $C_{\mathrm{SC}}$ and $C_{\mathrm{SS}}$ are given by ${ }^{12}$

$$
\begin{gathered}
C_{\mathrm{SC}} \equiv\left|d Q_{\mathrm{SC}} / d V_{S}\right|=(q / k T)\left|d Q_{\mathrm{SC}} / d \nu_{S}\right|, \\
C_{\mathrm{SS}} \equiv\left|d Q_{\mathrm{SS}} / d V_{S}\right|=(q / k T)\left|d Q_{\mathrm{SS}} / d \nu_{S}\right|,
\end{gathered}
$$

where $Q_{\mathrm{SC}}$ is the space-charge density, $Q_{\mathrm{SS}}$ is the surfacestate-charge density which is equal to $-q N_{\mathrm{SS}}$ for acceptorlike surface states and $+q N_{\mathrm{SS}}$ for donorlike surface states, $q$ is the electron charge, $k$ is the Boltzmann constant, $T$ is temperature, and $\nu_{S} \equiv q V_{S} / k T$ is dimensionless barrier height. For the case of fully ionized impurities and single-charge surface states characterized with both the density $N_{\mathrm{SS}}$ and effective energy level $E_{t}$, one yields ${ }^{12}$

$$
C_{\mathrm{SC}}=\left(\varepsilon \varepsilon_{0} / L\right)\left|d F_{S} / d v_{S}\right|, \quad C_{\mathrm{SS}}=\left(q^{2} N_{\mathrm{SS}} / k T\right)\left|d f\left(E_{t}\right) / d \nu_{S}\right|,
$$

where $\varepsilon$ and $\varepsilon_{0}$ are, respectively, the relative and vacuum dielectric constants of $\mathrm{GaN}, L$ is the effective Debye length characterizing the width of the space-charge region, $F_{S}$ is the value of a function $F$ at the surface, and $f\left(E_{t}\right)$ is the FermiDirac distribution function. Usually, the exact expression of the function $F$ for a metal-semiconductor Schottky contact is not easily found. A study of the characteristics of the functions $F_{S}$ and $f\left(E_{t}\right)$ reveals that $C_{\mathrm{SC}}$ achieves a minimum value in the depletion layers and increases rapidly in both accumulation and inversion layers. On the other hand, $C_{\mathrm{SS}}$ reaches its maximum value when the Fermi level at the surface crosses the effective energy level $E_{t}$ which is induced by the surface states. Based on the work of Many et al., ${ }^{12} C_{\mathrm{SS}}$ can be derived as 


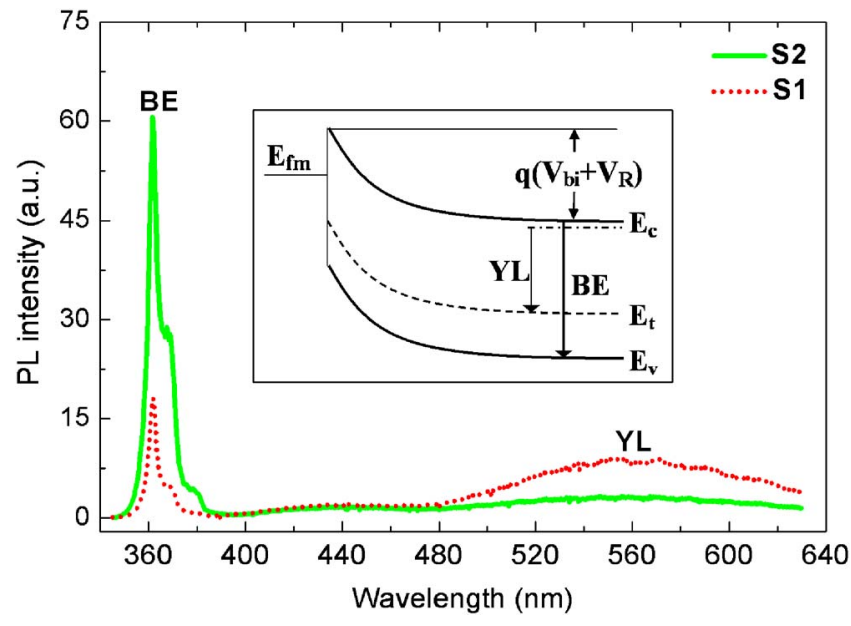

FIG. 3. (Color online) Room-temperature PL spectra of samples S1 and S2. The band-edge transition and yellow luminescence band are denoted by BE and YL, respectively. The inset depicts the band diagram of the $\mathrm{Au} / n$ GaN Schottky contact under reverse bias. $q V_{\text {bi }}$ and $q V_{R}$ are the barrier height at thermal equilibrium of the contact and the potential drop due to the reverse bias, respectively. $E_{\mathrm{fm}}$ represents the Fermi level of gold metal.

$$
C_{\mathrm{SS}}=\frac{q^{2} N_{\mathrm{SS}}}{k T} \frac{\exp \left[\left(E_{t}-E_{i}\right) / k T-u_{s}\right]}{\left\{1+\left[\exp \left(E_{t}-E_{i}\right) / k T-u_{s}\right]\right\}^{2}},
$$

where $E_{i}$ is the Fermi level of intrinsic semiconductor at $0 \mathrm{~K}$ and $u_{S}$ is the dimensionless surface potential which is related to $\nu_{S}$ in a relationship of $\nu_{S} \equiv u_{S}-u_{b}$. Here $u_{b}$ is a constant. Using Eqs. (2)-(6), we did a fitting to the $C$ - $V$ curve measured at $1 \mathrm{kHz}$ for the device fabricated on $\mathrm{S} 1$, as shown in Fig. 2. $N_{\mathrm{SS}}$ of the device fabricated on $\mathrm{S} 1$ is determined to be around $1.2 \times 10^{14} \mathrm{~cm}^{-2}$ and the effective energy level $E_{t}$ is $1.3 \mathrm{eV}$.

As the energy gaps of GaN epilayers range between 3.4 and $3.5 \mathrm{eV}$, the energy difference $\Delta$ between the defect level $E_{t}$ and the conduction band, i.e., $\Delta=E_{\text {gap }}-E_{t}$, is around $2.1-2.2 \mathrm{eV}$. This energy difference corresponds to the yellow band emission which has been widely assigned to the optical transition from the shallow donors (e.g., residual $\mathrm{Si}$ ) to the deep level centers. The room-temperature PL spectra of the samples were measured as shown in Fig. 3. As expected, the intensity of the yellow luminescence band $\left(I_{\mathrm{YL}}\right)$ of sample $\mathrm{S} 1$ is much stronger than that of S2 whose the band-edge transition at $3.44 \mathrm{eV}\left(I_{\mathrm{BE}}\right)$ is much stronger. The ratio $I_{\mathrm{YL}} / I_{\mathrm{BE}}$ of $\mathrm{S} 1$ is ten times higher than that of $\mathrm{S} 2$, which indicates that the density of deep level centers of S1 is much higher than that of S2. As mentioned earlier, sample S1 has higher background electron concentration and much lower mobility compared to $\mathrm{S} 2$. A recent investigation on the microstructural origin of the yellow emission of GaN epilayers reveals that the emission is closely related to edge dislocations. ${ }^{13}$ Such edge dislocations (more likely defect complexes) may well be the deep level centers as detected in the variable-frequency $C-V$ measurements. Incidentally, different acid solutions were used to clean the surface of S1. However, it is found that the surface treatment has no significant influence on the electrical properties of the Schottky devices. For example, the peak structure in the reverse-bias $C-V$ characteristic is not affected by the surface treatment.
This means that the deep level centers involved are pretty well distributed throughout the body of the GaN epilayer. The inset in Fig. 3 shows a schematic diagram of Schottky barrier with deep level centers under reverse bias. The bandedge $(\mathrm{BE})$ and yellow luminescence $(\mathrm{YL})$ transitions are also indicated schematically by vertical arrows. When a reverse bias is applied, the uniform Fermi level across the entire structure will be split into quasi-Fermi levels for electrons and holes. The energy level of the deep centers varies with respect to the Fermi level in the depletion region of the Schottky barrier where the bands are bent. Hence, the occupation of the level will also change. As a result, the capacitance of the contact structure will be affected. On the other hand, as the reverse bias is increased, more electrons can gain enough energy to pass through the barrier, resulting in an enhancement of the tunneling and recombination currents, and the reverse current thus becomes larger. ${ }^{14}$

In conclusion, Au Schottky contacts on different $n$-GaN epilayers were prepared and studied. The results show that the deep level centers play an important role in determining the electrical characteristics of the Schottky contacts. The density and energy level of the deep level centers can be extracted from the measured $C-V$ curves of the devices.

Support from RGC-CERG Grants, HKSAR, under Contract Nos HKU7036/03P and HKU7021/04P is acknowledged. Two of the authors (R.X.W. and S.J.X.) wish to thank S.-J. Xiong for his helpful discussion on the theoretical fitting and Y. Wang and J. Y. Dai for sharing their variablefrequency $C-V$ measurement system.

${ }^{1}$ S. Nakamura, S. Pearton, and G. Fasol, The Blue Laser Diode (Springer, Berlin, 2000).

${ }^{2}$ T. G. G. Maffeis, M. C. Simmonds, S. A. Clark, F. Peiro, P. Haines, and P. J. Parbrook, J. Appl. Phys. 92, 3179 (2002).

${ }^{3}$ K. Wang, R. X. Wang, S. Fung, C. D. Beling, X. D. Chen, Y. Huang, S. Li, S. J. Xu, and M. Gong, Mater. Sci. Eng., B 117, 21 (2005).

${ }^{4}$ P. Kozodoy, J. P. Ibbetson, H. Marchand, P. T. Fini, S. Keller, J. S. Speck, S. P. DenBaars, and U. K. Mishra, Appl. Phys. Lett. 73, 975 (1998).

${ }^{5}$ J. W. P. Hsu, M. J. Manfra, D. V. Lang, S. Richter, S. N. G. Chu, A. M. Sergent, R. N. Kleiman, L. N. Pfeiffer, and R. J. Molnar, Appl. Phys. Lett. 78, 1685 (2001)

${ }^{6}$ Y. C. Chou, D. Leung, I. Smorchkova, M. Wojtowicz, R. Grundbacher, L. Callejo, Q. Kan, R. Lai, P. H. Liu, D. Eng, and A. Oki, Microelectron. Reliab. 44, 1033 (2004).

${ }^{7}$ Z. Y. Liu, D. A. Saulys, and T. F. Kuech, Appl. Phys. Lett. 85, 4391 (2004).

${ }^{8}$ M. R. H. Khan, T. Detchprohm, P. Hacke, K. Hiramatsu, and N. Sawaki, J. Phys. D 28, 1169 (1995).

${ }^{9}$ R. X. Wang, S. J. Xu, S. Fung, C. D. Beling, K. Wang, S. Li, Z. F. Wei, T. J. Zhou, J. D. Zhang, Y. Huang, and M. Gong, Appl. Phys. Lett. 87, 031906 (2005).

${ }^{10}$ S. M. Sze, Physics of Semiconductor Devices, 2nd ed. (Wiley, New York, 1981), p. 264.

${ }^{11}$ E. H. Rhoderick, Metal-Semiconductor Contacts (Oxford University Press, Oxford, 1978), p. 142.

${ }^{12}$ A. Many, Y. Goldstein, and N. B. Grover, Semiconductor Surface (NorthHolland, Amsterdam, 1965), p. 220.

${ }^{13}$ D. G. Zhao, D. S. Jiang, H. Yang, J. J. Zhu, Z. S. Liu, S. M. Zhang, J. W. Liang, X. Li, X. Y. Li, and H. M. Gong, Appl. Phys. Lett. 88, 241917 (2006).

${ }^{14}$ R. X. Wang, S. J. Xu, A. B. Djurišić, C. D. Beling, C. K. Cheung, C. H. Cheung, S. Fung, D. G. Zhao, H. Yang, and X. M. Tao, Appl. Phys. Lett. 89, 033503 (2006). 